\title{
Rayleigh-Bénard Convection for Nanofluids for More Realistic Boundary Conditions (Rigid-Free and Rigid-Rigid) Using Darcy Model
}

\author{
Jyoti Ahuja \\ Department of Mathematics \\ Post Graduate Government College \\ Chandigarh, India \\ E-mail: jyotiahuja1985@gmail.com \\ Urvashi Gupta \\ Dr. S. S. Bhatnagar University Institute of Chemical Engineering \& Technology \\ Panjab University, Chandigarh, India \\ Corresponding author: dr_urvashi_gupta@yahoo.com; urvashi@pu.ac.in
}

(Received December 7, 2017; Accepted August 7, 2018)

\begin{abstract}
In this article, Rayleigh-Bénard convection for nanofluids for more realistic boundary conditions (rigid-free and rigidrigid) under the influence of the magnetic field is investigated. Presence of nanoparticles in base fluid has introduced one additional conservation equation of nanoparticles that incorporates the effect of thermophoretic forces and Brownian motion and the inclusion of magnetic field has introduced Lorentz's force term in the momentum equation along with Maxwell's equations. The solution of the Eigen value problem is found in terms of Rayleigh number by implementing the technique of normal modes and weighted residual Galerkin approximation. It is found that the stationary as well as oscillatory motions come into existence and heat transfer takes place through oscillatory motions. The critical Rayleigh number for alumina water nanofluid has an appreciable increase in its value with the rise in Chandrasekhar number and it increases moderately as we move from rigid-free to both rigid boundaries. The effect of different nanofluid parameters on the onset of thermal convection for two types of boundaries is investigated.
\end{abstract}

Keywords- Darcy’s law, Brownian motion, Magneto-convection, Chandrasekhar number.

\section{Introduction}

The thermal instability of a fluid layer in a porous medium has emerged as an evident problem considering its widespread usage across various utility applications such as enhanced oil recovery, storage of agricultural products, geothermal reservoirs and the underground pollutant transport. For Newtonian fluids, a comprehensive work on the problem of Rayleigh-Bénard convection has been done (Chandrasekhar, 1981) while considering different aspects of hydrodynamics and hydromagnetics. Nanofluid is a very prominent and significant term which is being highly discussed over a broad spectrum considering it's relevance and usage. A nanofluid is basically a fluid that is formed by suspending particles of nano-sized such as ceramics, metals, oxides, nitrides, and semiconductors in base fluids like water, ethylene glycol, oil etc. The usefulness of these suspensions is also revealed in the study (Choi, 1995) which also emphasizes the importance of nanofulids in enhancing the heat transfer mechanism because of exhibiting enormously high thermal conductivity. In continuation, other researchers (Das et al., 2003; Masuda et al., 1993) confirmed the same results through their experimental work. Due to enhanced thermal properties, it is now used in nuclear power plants (Buongiorno and $\mathrm{Hu}, 2005$ ) and in delivering drugs (Kleinstreuer et al., 2008). Further, convective instability of nanofluids is considered (Kim et al., 2004) and have shown that the onset of convection is hastened due to the 
International Journal of Mathematical, Engineering and Management Sciences

Vol. 4, No. 1, 139-156, 2019

https://dx.doi.org/10.33889/IJMEMS.2019.4.1-013

enhanced thermal conductivity of nanoparticles. (Buongiorno, 2006) suggested a model using the mechanics of nanoparticles and included the effects due to Brownian motion and thermophoretic diffusion. Buongiorno's model was utilized to analyze the thermal instability problems by many others (Tzou, 2008; Nield and Kuznetsov, 2009; Nield and Kuznetsov, 2010). In the past, the modified problem of thermal convection (Bhadauria and Agarwal, 2011; Yadav et al., 2011) is considered to include the impact of rotation.

Magneto-convection refers to the thermal convection under the effect of the applied magnetic field. According to the experimental results, (Heris et al., 2012) the nanoparticles concentration and the strong magnetic field increase the thermal efficiency of thermosyphon considerably. The impact of applied magnetic field/Hall currents on a layer of nanofluid has been studied (Gupta et al., 2013; 2014) and they have shown that the critical Rayleigh number along with critical wave number undergoes an appreciable rise with the rise in magnetic field parameter and considerable fall with the rise in Hall parameter. The authors considered the convection problem for the case of free-free boundaries, which are not realistic for the case of geophysical situations. The present formulation of the thermal convection problem introduces the impact of permeability and vertical magnetic field using the Darcy model for a porous medium for two types of boundaries: bothrigid and rigid-free. The Rayleigh number at which the instability sets in for alumina-water nanofluid has been identified under different boundaries. The problem has great application in geophysics due to the high magnetic field of the earth, its porous structure and owing to more realistic boundary conditions.

\section{Conservation Equations using Darcy Model}

In a homogenous porous medium, a thin layer of nanofluid (heated from below) is considered. Temperature and volume fraction of nanoparticles are $T_{h}$ and $\phi_{1}$ at the lower boundary $(z=0)$; and at the upper boundary $(z=d)$ is $T_{c}$ and $\phi_{0}\left(T_{h}>T_{c}, \phi_{1}>\phi_{0}\right)$ as in Figure 1 . The gravitational force $\boldsymbol{g}=(0,0,-g)$ and the magnetic field $\boldsymbol{H}=(0,0, H)$ are acting in the opposite directions. In the porous medium Darcy model (Nield and Kuznetsov, 2009) is incorporated to analyze the stability of the system. The Darcy velocity is denoted by ' $\boldsymbol{v}_{D}$ ' i.e. $\boldsymbol{v}_{D}=\varepsilon \boldsymbol{q}$ where $\boldsymbol{q}$ is the velocity of nanofluid and $\varepsilon$ denotes the porosity of the medium. 
International Journal of Mathematical, Engineering and Management Sciences

Vol. 4, No. 1, 139-156, 2019

https://dx.doi.org/10.33889/IJMEMS.2019.4.1-013

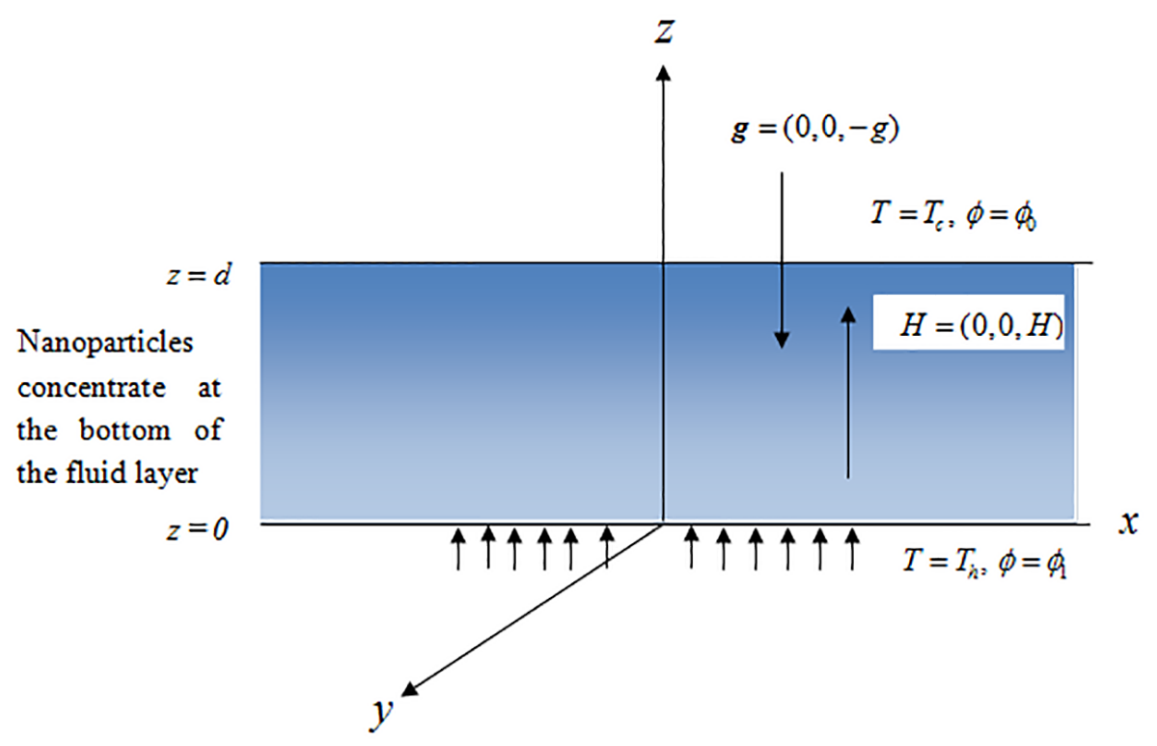

Figure 1. Sketch of the physical system

The hydrodynamic and hydromagnetic equations using the Darcy model and the assumptions as discussed by (Gupta et al., 2013) are

$\nabla \cdot \boldsymbol{v}_{D}=0$,

$\frac{\partial \phi}{\partial t}+\nabla \phi \cdot \frac{v_{D}}{\varepsilon}=\nabla \cdot\left[D_{T} \frac{\nabla T}{T}+\nabla \phi \cdot D_{B}\right]$,

$0=\tilde{\mu} \nabla^{2} \boldsymbol{v}_{D}-\nabla p+\rho \boldsymbol{g}-\frac{\mu}{k_{1}} \boldsymbol{v}_{D}+\frac{\mu_{e}}{4 \pi}(\nabla \times \boldsymbol{h}) \times \boldsymbol{H}$,

$v_{D} \cdot \nabla T(\rho c)_{f}+(\rho c)_{m} \frac{\partial T}{\partial t}=\left(k_{m} \nabla^{2} T\right)+\varepsilon(\rho c)_{p}\left[D_{T} \frac{\nabla T . \nabla T}{T}+D_{B} \nabla T . \nabla \phi\right]$,

The density of nanofluid is denoted by $\rho$ which is given as

$\rho=\phi \rho_{p}+(1-\phi) \rho_{f}, \cong \phi \rho_{p}+(1-\phi)\left\{\rho_{0}\left(1-\beta\left(T-T_{0}\right)\right)\right\}$,

and Maxwell's equations are

$\frac{d \boldsymbol{h}}{d t}=(\boldsymbol{H} \cdot \nabla) \frac{\boldsymbol{v}_{D}}{\varepsilon}+\eta \nabla^{2} \boldsymbol{h}$,

$\nabla . \boldsymbol{h}=0$,

where $\eta$ is the resistivity of the fluid. In order to get the non-dimensional parameter thermal Rayleigh number the above equations are non-dimensionalized by using 
International Journal of Mathematical, Engineering and Management Sciences

Vol. 4, No. 1, 139-156, 2019

https://dx.doi.org/10.33889/IJMEMS.2019.4.1-013

$\left(x^{\prime}, y^{\prime}, z^{\prime}\right)=(x, y, z) / d, t^{\prime}=t \alpha_{m} / \sigma d^{2}, \quad h^{\prime}=\left(\eta / H \alpha_{m}\right) h,\left(u^{\prime}, v^{\prime}, w^{\prime}\right)=(u, v, w) d / \alpha_{m}, \quad p^{\prime}=p k_{1} / \mu \alpha_{m}$,

$\phi^{\prime}=\left(\phi-\phi_{0}\right) /\left(\phi_{1}-\phi_{0}\right), \quad T^{\prime}=\left(T-T_{c}\right) /\left(T_{h}-T_{c}\right)$, where $\alpha_{m}=k_{m} /(\rho c)_{f}, \sigma=(\rho c)_{m} /(\rho c)_{f}$.

After dropping the dashes, Eqs. (1)-(7) are transformed into

$\nabla . \mathbf{q}=0$,

$0=-\nabla p-\boldsymbol{q}-R_{m} \hat{e}_{z}-R_{c n} \phi \hat{e}_{z}+R_{a} T \hat{e}_{z}+Q\left[\left(\frac{\partial h_{x}}{\partial z}-\frac{\partial h_{z}}{\partial x}\right) \hat{e}_{x}-\left(\frac{\partial h_{z}}{\partial y}-\frac{\partial h_{y}}{\partial z}\right) \hat{e}_{y}\right]$,

$\frac{\partial T}{\partial t}+\boldsymbol{q} \cdot \nabla T=\nabla^{2} T+\frac{N_{B}}{L_{h}}(\nabla \phi . \nabla T)+\frac{N_{A} N_{B}}{L_{h}}(\nabla T . \nabla T)$,

$\frac{1}{\sigma} \frac{\partial \phi}{\partial t}+\frac{1}{\varepsilon} \boldsymbol{q} \cdot \nabla \phi=\frac{1}{L_{h}} \nabla^{2} \phi+\frac{N_{A}}{L_{h}} \nabla^{2} T$,

$\nabla \cdot \boldsymbol{h}=0$,

$\frac{\varepsilon}{\sigma} \frac{\operatorname{Pr}_{2}}{\operatorname{Pr}_{1}} \frac{d \boldsymbol{h}}{d t}=\left(\frac{\partial u}{\partial z} \hat{e}_{x}+\frac{\partial v}{\partial z} \hat{e}_{y}+\frac{\partial w}{\partial z} \hat{e}_{z}\right)+\varepsilon \nabla^{2} \boldsymbol{h}$,

where

$\operatorname{Pr}_{1}=\mu / \rho \alpha_{m}:($ the Prandtl number $)$;

$N_{A}=\left(D_{T}\left(T_{h}-T_{c}\right)\right) /\left(D_{B} T_{c}\left(\phi_{1}-\phi_{0}\right)\right):$ the modified diffusivity ratio;

$\operatorname{Pr}_{2}=\mu / \rho \eta$ : the magnetic Prandtl number ;

$L_{h}=\alpha_{m} / D_{B}:$ the Lewis number;

$R_{a}=\left(\rho g \beta d k_{1}\left(T_{h}-T_{c}\right)\right) / \mu \alpha_{m}:$ the Rayleigh number;

$N_{B}=\left[\varepsilon(\rho c)_{p} /(\rho c)_{f}\right]\left(\phi_{1}-\phi_{0}\right):$ the modified particle-density increment;

$R_{m}=\left[\phi_{0} \rho_{p}+\rho\left(1-\phi_{0}\right) / \mu \alpha_{m}\right] g d k_{1}:$ the basic density Rayleigh number;

$R_{c n}=\left(\left(\rho_{p}-\rho\right)\left(\phi_{1}-\phi_{0}\right) / \mu \alpha_{m}\right) g d k_{1}$ : the concentration Rayleigh number;

and $Q=\left(\mu_{e} H^{2} k_{1} / 4 \pi \eta \mu\right)$ : the Chandrasekhar number.

\section{Initial Flow and Perturbation Equations}

The initial flow is supposed to be stationary and the temperature, nanoparticle fraction and pressure are assumed to vary in $z$ direction only i.e.

$\boldsymbol{q}=0, T=T_{\mathrm{I}}(z), p=p_{\mathrm{I}}(z), \phi=\phi(z)$.

The subscript 'I' represents the initial flow. Applying Eq.(16) to Eqs. (10)-(12) and integrating w.r.t. $z$, we get

$\phi=-N_{A} T_{I}+\left(1+N_{A}\right)(1-z)$, 
International Journal of Mathematical, Engineering and Management Sciences

Vol. 4, No. 1, 139-156, 2019

https://dx.doi.org/10.33889/IJMEMS.2019.4.1-013

$T_{\mathrm{I}}=\frac{1-e^{-\left(1+N_{A}\right) N_{B}(1-z) / L_{h}}}{1-e^{-\left(1+N_{A}\right) N_{B} / L_{h}}}$.

As suggested by Buongiorno (2006), $L_{h}$ is large and $N_{A}$ is small. By neglecting the second and higher order terms, Eqs. (18) and (17) reduce to

$T_{\mathrm{I}}=1-z$,

$\phi_{1}=1-z$.

Let us apply very small perturbations (denoted by bar symbol) on the initial flow

$\boldsymbol{q}(u, v, w)=0+\overline{\boldsymbol{q}}, \phi=\phi_{\mathrm{I}}+\bar{\phi}, p=p_{\mathrm{I}}+\bar{p}, \boldsymbol{h}\left(h_{x}, h_{y}, h_{z}\right)=\boldsymbol{H}+\overline{\boldsymbol{h}}, T=T_{\mathrm{I}}+\bar{T}$,

with $T_{\mathrm{I}}=\phi_{\mathrm{I}}=1-z$. Applying (21) to Eqs. (9)-(14), linearizing and using Eqs. (19) and (20), we get

$\nabla \cdot \boldsymbol{q}=0$,

$\nabla^{2} w-Q \nabla^{2} \frac{\partial h_{z}}{\partial z}+R_{c n} \nabla_{H}^{2} \phi-R_{a} \nabla_{H}^{2} T=0$,

$-w+\frac{\partial T}{\partial t}=\nabla^{2} T-2 \frac{N_{A} N_{B}}{L_{h}} \frac{\partial T}{\partial z}-\frac{N_{B}}{L_{h}}\left(\frac{\partial \phi}{\partial z}+\frac{\partial T}{\partial z}\right)$,

$\frac{1}{\sigma} \frac{\partial \phi}{\partial t}-\frac{w}{\varepsilon}=\frac{1}{L_{h}} \nabla^{2} \phi+\frac{N_{A}}{L_{h}} \nabla^{2} T$,

$\varsigma-Q \frac{\partial \xi}{\partial z}=0$

$\frac{\varepsilon}{\sigma} \frac{\operatorname{Pr}_{2}}{\operatorname{Pr}_{1}} \frac{\partial \xi}{\partial t}=\frac{\partial \varsigma}{\partial z}+\varepsilon \nabla^{2} \xi$

$\frac{\varepsilon}{\sigma} \frac{\operatorname{Pr}_{2}}{\operatorname{Pr}_{1}} \frac{\partial h_{z}}{\partial t}-\varepsilon \nabla^{2} h_{z}=\frac{\partial w}{\partial z}$

where $\nabla_{H}^{2}=\partial^{2} / \partial x^{2}+\partial^{2} / \partial y^{2}$ and $\nabla p$ is eliminated from Eqs.(23), (27) and (28) by introducing $\varsigma=(\partial v / \partial x-\partial u / \partial y)$ and $\xi=\left(\partial h_{y} / \partial x-\partial h_{x} / \partial y\right)$.The system of differential Eqs. (22)-(28) will be used in further analysis by applying the methodology of superposition of basic modes and one term Galerkin residual approximation.

\section{Normal Mode Analysis and Galerkin Type Weighted Residual Method}

In the present study, we have used the methodology of superposition of basic modes in which the perturbed quantities are expressed as

$$
\left(w, \xi, T, \zeta, \phi, h_{z},\right)=(W(z), X(z), \Theta(z), Z(z), \Phi(z), K(z)) \exp \left(i l_{x} x+i l_{y} y+n t\right)
$$


International Journal of Mathematical, Engineering and Management Sciences

Vol. 4, No. 1, 139-156, 2019

https://dx.doi.org/10.33889/IJMEMS.2019.4.1-013

where $l_{x}$ and $l_{y}$ are the wave numbers in two directions (horizontal and vertical) and $n$ is the growth rate. The linearized equations after the process of elimination are

$$
\begin{aligned}
& \left(D^{2}-a^{2}\right) W-Q\left(D^{2}-a^{2}\right) D K-R_{c n} a^{2} \Phi+R_{a} a^{2} \Theta=0, \\
& \left\{\frac{\varepsilon}{\sigma} \frac{\operatorname{Pr}_{2}}{\operatorname{Pr}_{1}} n-\varepsilon\left(D^{2}-a^{2}\right)\right\} K=D W, \\
& n \Theta-W-\left(D^{2}-\frac{N_{B}}{L_{n}} D-2 \frac{N_{A} N_{B}}{L_{n}} D-a^{2}\right) \Theta+\frac{N_{B}}{L_{n}} D \Phi=0, \\
& \frac{1}{\sigma} n \Phi-\frac{W}{\varepsilon}-\frac{N_{A}}{L_{n}}\left(D^{2}-a^{2}\right) \Theta-\frac{1}{L_{n}}\left(D^{2}-a^{2}\right) \Phi=0,
\end{aligned}
$$

where $D \equiv d / d z$ and $a=\left(l_{x}^{2}+l_{y}^{2}\right)^{1 / 2}$ is the resultant wave number. Eliminating $K$ from Eqs. (30) and (31), we obtain

$$
\left[\left\{\left(D^{2}-a^{2}\right)\right\}\left\{\frac{\varepsilon}{\sigma} \frac{\operatorname{Pr}_{2}}{\operatorname{Pr}_{1}} n-\varepsilon\left(D^{2}-a^{2}\right)\right\}-Q D^{2}\left(D^{2}-a^{2}\right)\right] W+\left\{\frac{\varepsilon}{\sigma} \frac{\operatorname{Pr}_{2}}{\operatorname{Pr}_{1}} n-\varepsilon\left(D^{2}-a^{2}\right)\right\}\left(R_{a} a^{2} \Theta-R_{c n} a^{2} \Phi\right)=0 .
$$

Now in order to solve Eqs. (32)-(34), Galerkin weighted residuals approach is adopted. Choice of the trial functions $W_{p}, \Theta_{p}$ and $\Phi_{p}$ depends on the relevant boundary conditions and we write

$$
W=\sum_{p=1}^{N} A_{p} W_{p}, \quad \Theta=\sum_{p=1}^{N} B_{p} \Theta_{p}, \quad \Phi=\sum_{p=1}^{N} C_{p} \Phi_{p}
$$

For single term approximation, we put $p=1$. After substituting Eq. (35) in Eqs. (32)-(34) and making use of orthogonality; we obtain a system of three equations in three unknowns $A_{1}, B_{1}, C_{1}$. Elimination of these unknowns from the obtained set of equations gives the Eigen value equation.

\section{Results and Discussion}

\subsection{Rigid-Rigid Boundaries}

Let us now consider that both the boundaries of the fluid layer are rigid. Therefore, the vanishing of the normal as well as horizontal components of the velocity to the rigid surface (no slip condition) leads to the boundary conditions

$W=0, \Phi=0, \Theta=0$ and $D W=0$, at both the boundaries $K=0, D K=0$,

for perfectly conducting boundaries. The suitable trial functions satisfying this boundary condition are

$$
\Theta_{1}=z(1-z), W_{1}=z^{2}\left(1-z^{2}\right), \Phi_{1}=z(1-z) .
$$


International Journal of Mathematical, Engineering and Management Sciences

Vol. 4, No. 1, 139-156, 2019

https://dx.doi.org/10.33889/IJMEMS.2019.4.1-013

Using the approximation of single term Galerkin residual method and orthogonality, the eigen value equation becomes

$$
\begin{aligned}
& {\left[-\frac{\varepsilon}{\sigma} \frac{\operatorname{Pr}_{2}}{\operatorname{Pr}_{1}} n\left\{\frac{2}{105}+\frac{a^{2}}{630}\right\}-\varepsilon\left\{\frac{24}{30}+4 \frac{a^{2}}{105}+\frac{a^{4}}{630}\right\}-Q\left\{\frac{2}{105}+\frac{2 a^{2}}{105}\right\}\right]\left[140\left(\frac{n}{30}+\frac{a^{2}}{30}+\frac{1}{3}\right)\right]} \\
& {\left[\frac{n}{\sigma} \cdot \frac{1}{30}+\left(\frac{a^{2}}{30}+\frac{1}{3}\right) \frac{1}{L_{n}}\right]+\left[\frac{\varepsilon}{\sigma} \frac{\operatorname{Pr}_{2}}{\operatorname{Pr}_{1}} n \cdot \frac{1}{140}+\varepsilon\left\{\frac{2}{30}+\frac{a^{2}}{140}\right\}\right]\left[R_{a} a^{2}\left(\frac{n}{\sigma} \cdot \frac{1}{30}\right)+\left(\frac{a^{2}}{30}+\frac{1}{3}\right) \frac{1}{L_{n}}\right]} \\
& -R_{c n} a^{2}\left[\frac{1}{\varepsilon}\left(\frac{n}{30}+\frac{a^{2}}{30}+\frac{1}{3}\right)-\left(\frac{a^{2}}{30}+\frac{1}{3}\right) \frac{N_{A}}{L_{n}}\right]=0 .
\end{aligned}
$$

Let us discuss the case of stationary convection by putting $n=0$ in Eq. (38). The expression for Rayleigh number for both rigid boundaries become

$$
R_{a}(\text { stat })=R_{c n}\left(\frac{L_{n}}{\varepsilon}-N_{A}\right)+\frac{140}{a^{2}\left(\frac{2}{30}+\frac{a^{2}}{140}\right)}\left[\left\{\frac{504+24 a^{2}+a^{4}}{630}\right\}+\frac{Q}{\varepsilon}\left\{\frac{168+4 a^{2}}{210}\right\}\right]\left(\frac{a^{2}}{30}+\frac{1}{3}\right)
$$

Critical Rayleigh number along with critical wave number is found by the condition $d R_{a} / d a=0$, numerically. In the absence of nanoparticles and magnetic field the value of critical wave number is $a_{c}=4.791$ and critical Rayleigh number is 72.94 . For oscillatory convection we put $n=i \omega \neq 0$ in the Eigen value Eq. (38), and separate the imaginary and real parts to get an expression for $R_{a}(o s c)$. This expression is quite lengthy so we have written it in Appendix B1; nevertheless the same is used to plot the graphs of $R_{a}(o s c)$ for rigid-rigid boundaries.

\subsection{Rigid-Free Boundaries}

Let us now consider the lower boundary of the fluid to be rigid and the upper as free. The relevant boundary conditions on $W$ are

$D W=0, W=0$, at $z=0$ for lower boundary,

$D^{2} W=0, W=0$, at $z=1$ for upper boundary.

Trial function appropriate to these boundaries is

$W_{1}=z^{2}(1-z)(3-2 z)$.

By making use of single term Galerkin method and orthogonality, the Eigen value equation in the present case becomes 
International Journal of Mathematical, Engineering and Management Sciences

Vol. 4, No. 1, 139-156, 2019

https://dx.doi.org/10.33889/IJMEMS.2019.4.1-013

$$
\begin{aligned}
& \frac{420}{13}\left[-\frac{\varepsilon}{\sigma} \frac{\operatorname{Pr}_{2}}{\operatorname{Pr}_{1}} n\left\{\frac{12}{35}+a^{2} \frac{76}{2520}\right\}-\varepsilon\left\{\frac{36}{5}+\frac{24 a^{2}}{35}+a^{4} \frac{76}{2520}\right\}-Q\left\{\frac{36}{5}+\frac{12 a^{2}}{35}\right\}\right] \\
& \left(\frac{a^{2}}{30}+\frac{1}{3}+\frac{n}{30}\right)\left(\frac{1}{L_{n}}\left(\frac{a^{2}}{30}+\frac{1}{3}\right)+\frac{n}{30 \sigma}\right)+\left\{\frac{\varepsilon}{\sigma} \frac{\operatorname{Pr}_{2}}{\operatorname{Pr}_{1}} n \frac{13}{420}+\varepsilon\left\{\frac{3}{10}+\frac{13}{420} a^{2}\right\}\right\} \\
& {\left[R_{a} a^{2}\left(\frac{a^{2}}{30}+\frac{1}{3}+\frac{n}{30 \sigma}\right)-R_{c n} a^{2}\left\{\frac{1}{\varepsilon}\left(\frac{a^{2}}{30}+\frac{1}{3}+\frac{n}{30}\right)-\frac{N_{A}}{L_{n}}\left(\frac{a^{2}}{30}+\frac{1}{3}\right)\right\}\right]=0 .}
\end{aligned}
$$

For stationary convection, the Eigen value Eq. (43) takes the form

$$
R_{a}(\text { stat })=R_{c n}\left(\frac{L_{n}}{\varepsilon}-N_{A}\right)+\frac{420}{a^{2}\left(126+13 a^{2}\right)}\left[\left\{\frac{4536+432 a^{2}+19 a^{4}}{630}\right\}+\frac{Q}{\varepsilon}\left\{\frac{252+12 a^{2}}{35}\right\}\right]\left(\frac{a^{2}}{30}+\frac{1}{3}\right) \frac{420}{13}
$$

Equation (44) attains its minimum value when $a_{c}=3.97$ and this minimum is 56.97 (taking $Q=0, R_{c n}=0$ ). For oscillatory modes, the expression for Rayleigh number is found as given in Appendix B2 and the graphs are plotted using the software Mathematica.

Let us now analyze the critical values for alumina-water nanofluid $\left(L_{n}=5000, N_{A}=5\right.$ and fixed $\left.R_{c n}=0.1\right)$ for the two types of boundaries. It has been observed that as the porosity increases there is a fall in the critical wave number and a predominant fall in the critical Rayleigh number for the two type of boundaries (Table 1). This means as the ratio of pore space to total space increases the nanofluid layer system gets strongly destabilized and it hastens the onset of instability. As we move from rigid-free boundaries to both rigid boundaries $a_{c}$ shows a significant increase along with some increase in $R_{c}$ i.e. $\left(R_{c}\right)_{b o t h-\text { rigid }}>\left(R_{c}\right)_{\text {rigid-free }}$ which means that the stability of the fluid layer system increases as we move from rigid-free and both-rigid boundaries.

Table 1. Effect of $\varepsilon$ for alumina-water nano-fluid on $a_{c}$ and $R_{c}$ for $Q=100, R_{c n}=0.1$.

\begin{tabular}{ccccc}
\hline & Rigid-Free & \multicolumn{2}{c}{ Both Rigid } \\
\hline$\varepsilon$ & $a_{c}$ & $R_{c}$ & $a_{c}$ & $R_{c}$ \\
\hline 0.2 & 18.67 & 9219.44 & 22.52 & 9798.28 \\
0.4 & 15.71 & 4773.57 & 18.95 & 5130.24 \\
0.6 & 14.20 & 3268.28 & 17.13 & 3540.69 \\
0.8 & 13.22 & 2506.61 & 15.95 & 2732.95 \\
\hline
\end{tabular}


International Journal of Mathematical, Engineering and Management Sciences

Vol. 4, No. 1, 139-156, 2019

https://dx.doi.org/10.33889/IJMEMS.2019.4.1-013

Table 2. Effect of $Q$ for alumina-water nanofluid on $a_{c}$ and $R_{c}$ for $\varepsilon=0.7, R_{c n}=0.1$.

\begin{tabular}{lllll}
\hline & Rigid-Free & \multicolumn{3}{c}{ Both Rigid } \\
\hline$Q$ & $a_{c}$ & $R_{c}$ & $a_{c}$ & $R_{c}$ \\
\hline 0 & 3.97 & 770.97 & 4.791 & 786.94 \\
100 & 13.67 & 2834.25 & 16.49 & 3080.77 \\
500 & 20.41 & 10132.62 & 24.61 & 10884.2 \\
1000 & 24.26 & 19014.7 & 29.24 & 20292.3 \\
\hline
\end{tabular}

In Table 2, $a_{c}$ and $R_{c}$ are considered for different values of $Q$ and for $\varepsilon=0.7, R_{c n}=0.1$. Both $a_{c}$ and $R_{c}$ show a tremendous rise with the rise in magnetic field parameter for the two type of boundary conditions. Further $a_{c}$ and $R_{c}$ exhibit an increase while moving from rigid-free to both rigid boundaries. This shows that magnetic field bears a strong influence on the nanofluid layer system by way of increasing its stability and delaying the onset of thermal convection.

\section{Numerical Results and Discussion}

To analyse the behavior of parameters like $R_{c n}, Q, \varepsilon, L_{n}, N_{A}$ and $\sigma$ on the onset of convection; computations are carried out using Eqs. (39) and (44) for stationary convection for both-rigid and rigid-free boundaries, respectively. To carry out the computations in respect of oscillatory

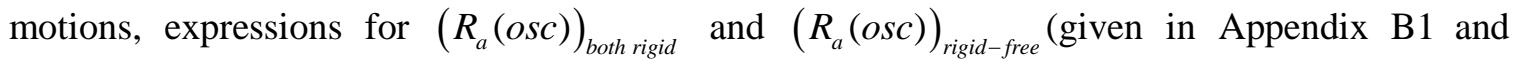
Appendix B2 as B.1.1 and B.1.2) are used for rigid-rigid and rigid-free boundaries, respectively. The curves representing the variation of $R_{a}($ stat $)$ and $R_{a}($ osc $)$ w.r.t the wave number ' $a$ ' for fixed values of other parameters have been sketched. The fixed values of various parameters are taken as $\operatorname{Pr}_{1}=1, \operatorname{Pr}_{2}=0.01, R_{c n}=4, L_{n}=100, Q=50, N_{A}=4, \varepsilon=0.7, \sigma=1$. By changing the value of one parameter the effect of that particular parameter can be examined on the convection problem.

Figures 2-11 show the neutral stability curves for non-oscillatory and oscillatory motions in porous medium for bottom heavy configuration of nanoparticles. Figure 2 is a plot of $R_{a}$ (stat) and Figure 3 is a plot of $R_{a}$ (osc) versus wave number for $R_{c n}=4,7$ and 10 . It is seen that $\left(R_{a}\right)_{\text {both rigid }}>\left(R_{a}\right)_{\text {rigid-free }}$ for both types of convections. If we compare Figures 2 and 3 it is easy to recognize that $R_{a}($ stat $)>R_{a}(\mathrm{osc})$ for fixed ' $a$ ' that means the mode of heat transfer is through oscillatory motions instead of stationary convection. Figure 2 presents a clear picture of the significant increase in $R_{a}$ (stat) with the rise in $R_{c n}$ whereas there is no significant increase in $R_{a}$ (osc) (Figure 3). Thus the increase in the concentration of nanoparticles makes the stationary convection much more stable as compared to over stable convection (Figure 3) when particle density decreases upward. 
International Journal of Mathematical, Engineering and Management Sciences

Vol. 4, No. 1, 139-156, 2019

https://dx.doi.org/10.33889/IJMEMS.2019.4.1-013

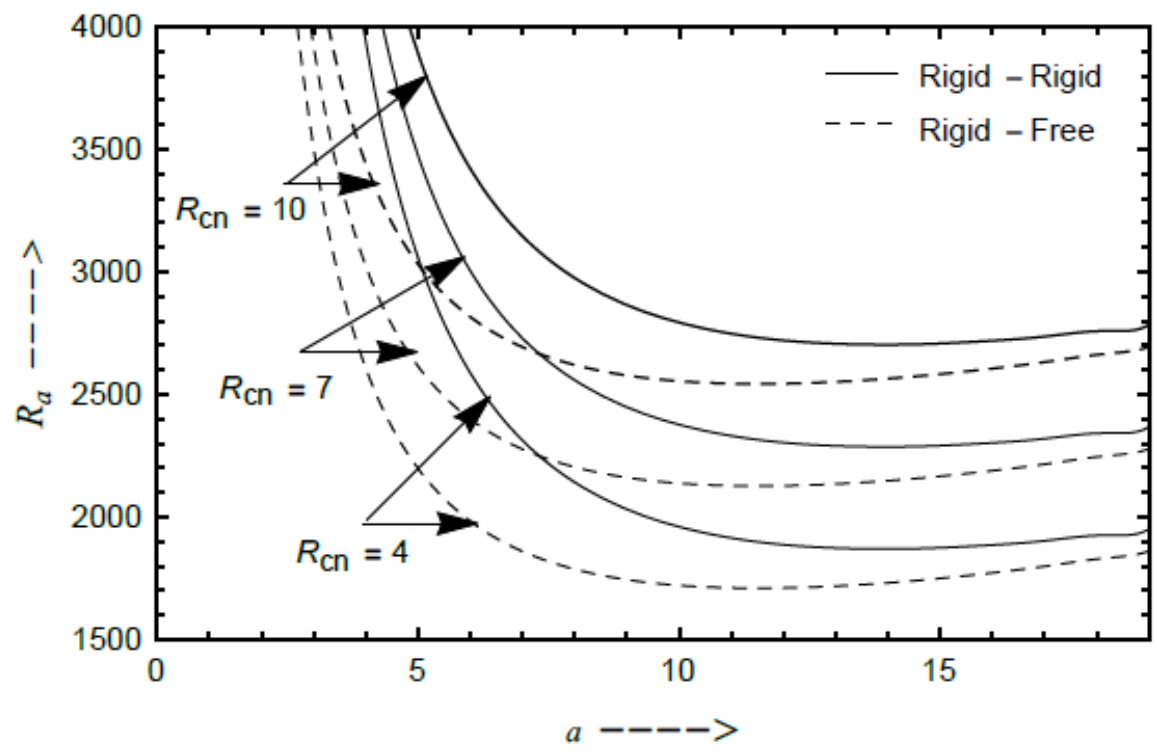

Figure 2. Influence of concentration Rayleigh number on $R_{a}$ (stat) for $R_{c n}=4,7$ and 10 for two different boundaries

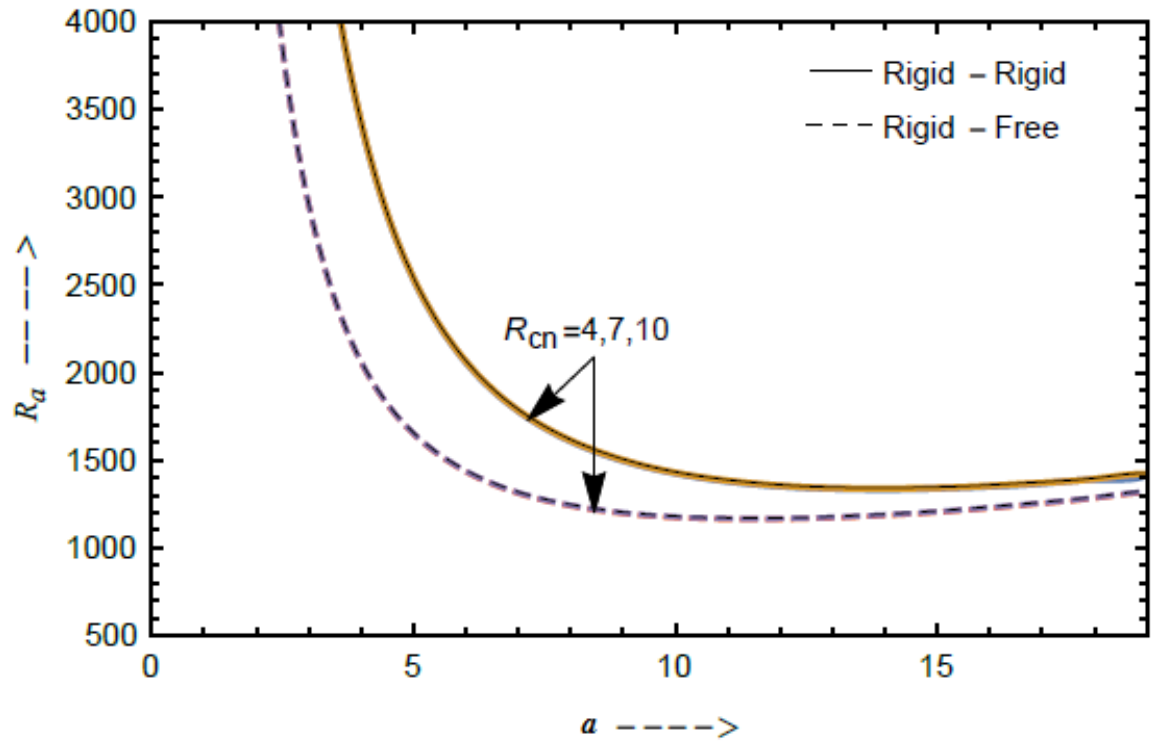

Figure 3. Influence of concentration Rayleigh number on $R_{a}$ (osc) for $R_{c n}=4,7$ and 10 for two different boundaries

Figures 4-5 correspond to Rayleigh numbers $R_{a}($ stat $)$ and $R_{a}(\mathrm{osc})$, respectively, for $Q=50,100$ and 150 . Both these figures show that there is a remarkable enhancement in the 
International Journal of Mathematical, Engineering and Management Sciences

Vol. 4, No. 1, 139-156, 2019

https://dx.doi.org/10.33889/IJMEMS.2019.4.1-013

values of $R_{a}$ (stat) as well as $R_{a}$ (osc) with the increase in Chandrasekhar number. Thus, the application of magnetic field postpones the onset of convection and this delaying effect grows in magnitude with the rise in the magnetic field. Further, it is interpreted from the figures that the curves showing the effect of the magnetic field for rigid-free boundaries, lies below the curves for both rigid boundaries. The fluid layer confined between both rigid boundaries is more stable as compared to rigid-free boundaries. Figure 4-5 show that $R_{a}($ stat $)>R_{a}$ (osc) for all values of the Chandrasekhar number maintaining the fact that oscillatory motions prevail the system.

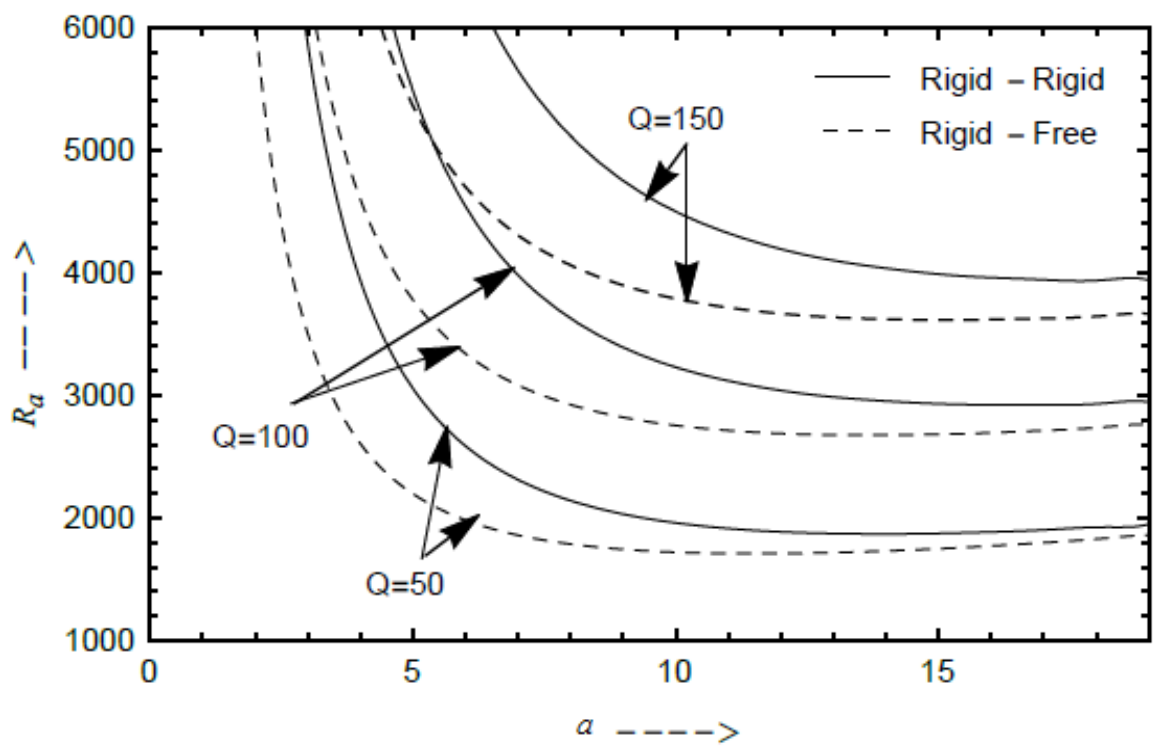

Figure 4. Influence of magnetic field on $R_{a}$ (stat) for $Q=50,100$ and 150 for two different boundaries

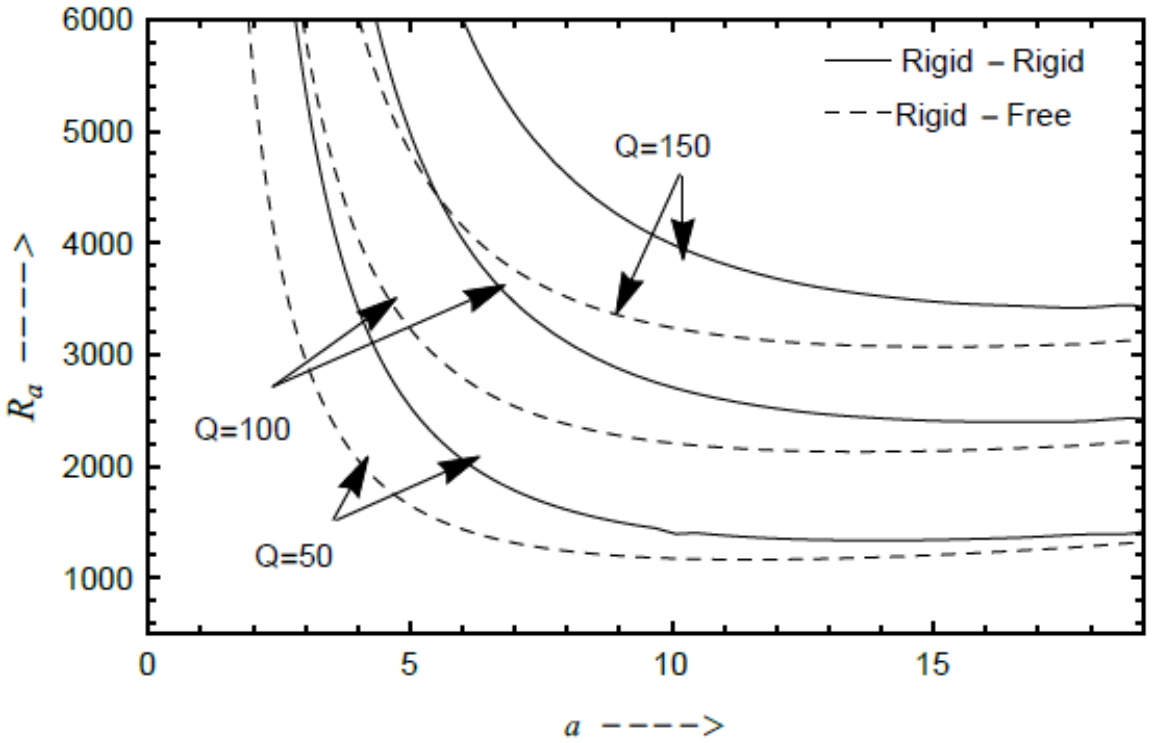

Figure 5. Influence of magnetic field on $R_{a}(\mathrm{osc})$ for $Q=50,100$ and 150 for two different boundaries. 
International Journal of Mathematical, Engineering and Management Sciences

Vol. 4, No. 1, 139-156, 2019

https://dx.doi.org/10.33889/IJMEMS.2019.4.1-013

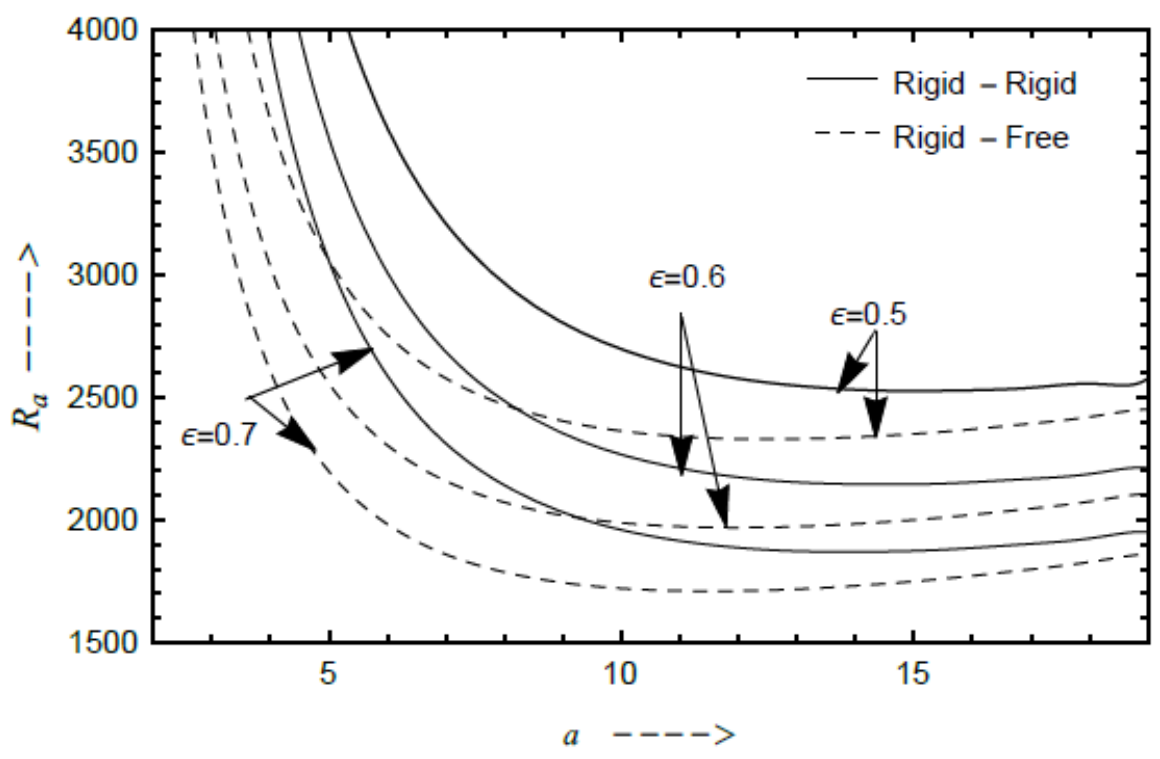

Figure 6. Influence of porosity on $R_{a}($ stat $)$ for $\varepsilon=0.5,0.6$ and 0.7 for two different boundaries

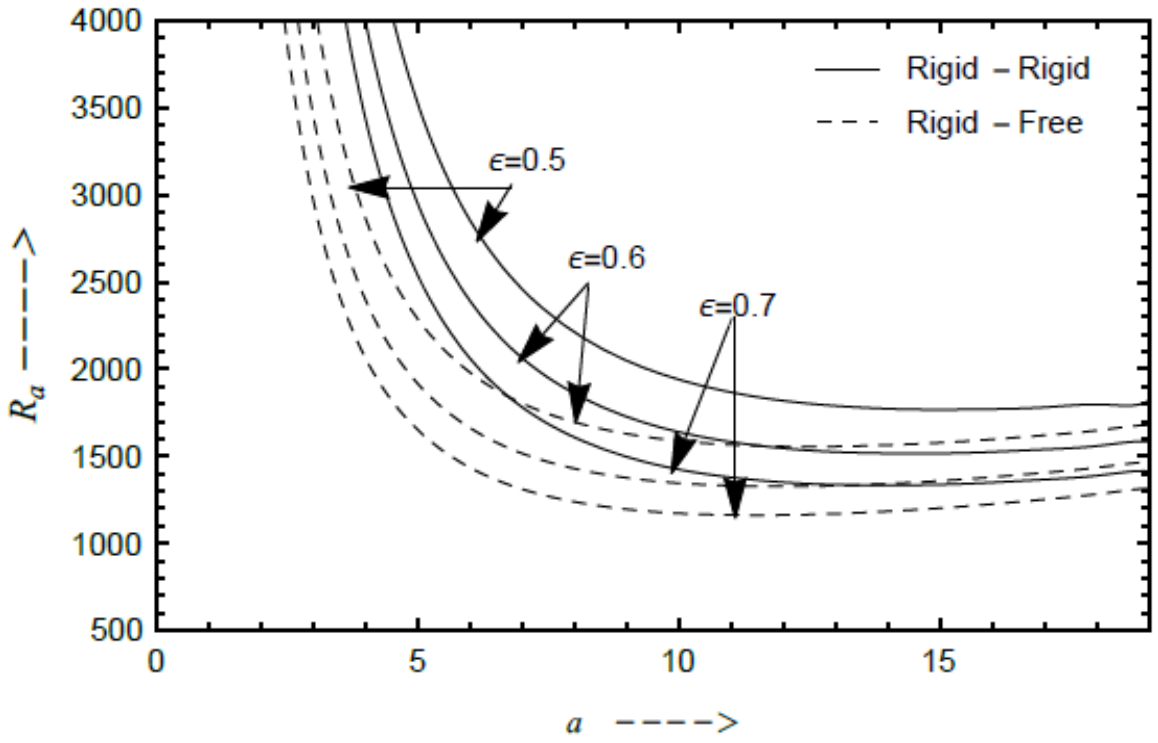

Figure 7. Influence of porosity on $R_{a}(\mathrm{osc})$ for $\varepsilon=0.5,0.6$ and 0.7 for two different boundaries 
International Journal of Mathematical, Engineering and Management Sciences

Vol. 4, No. 1, 139-156, 2019

https://dx.doi.org/10.33889/IJMEMS.2019.4.1-013

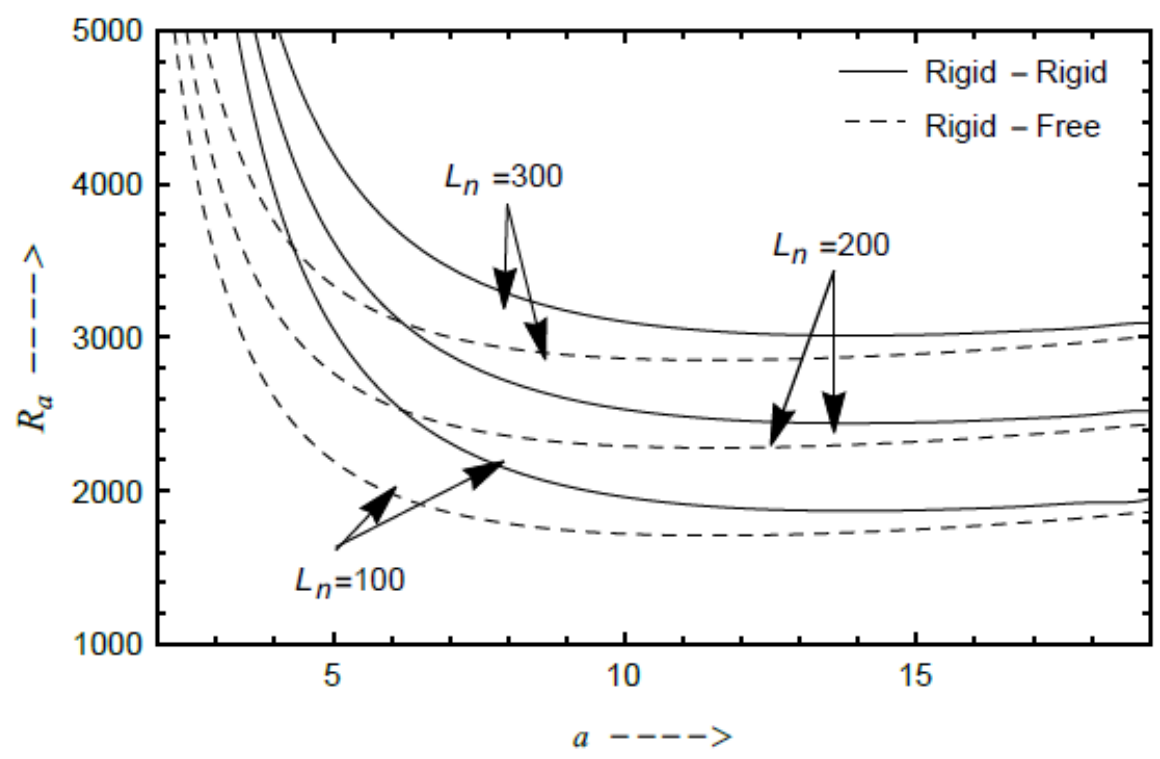

Figure 8. Influence of Lewis number on $R_{a}($ stat $)$ for $L_{n}=100,200$ and 300 for three different boundaries

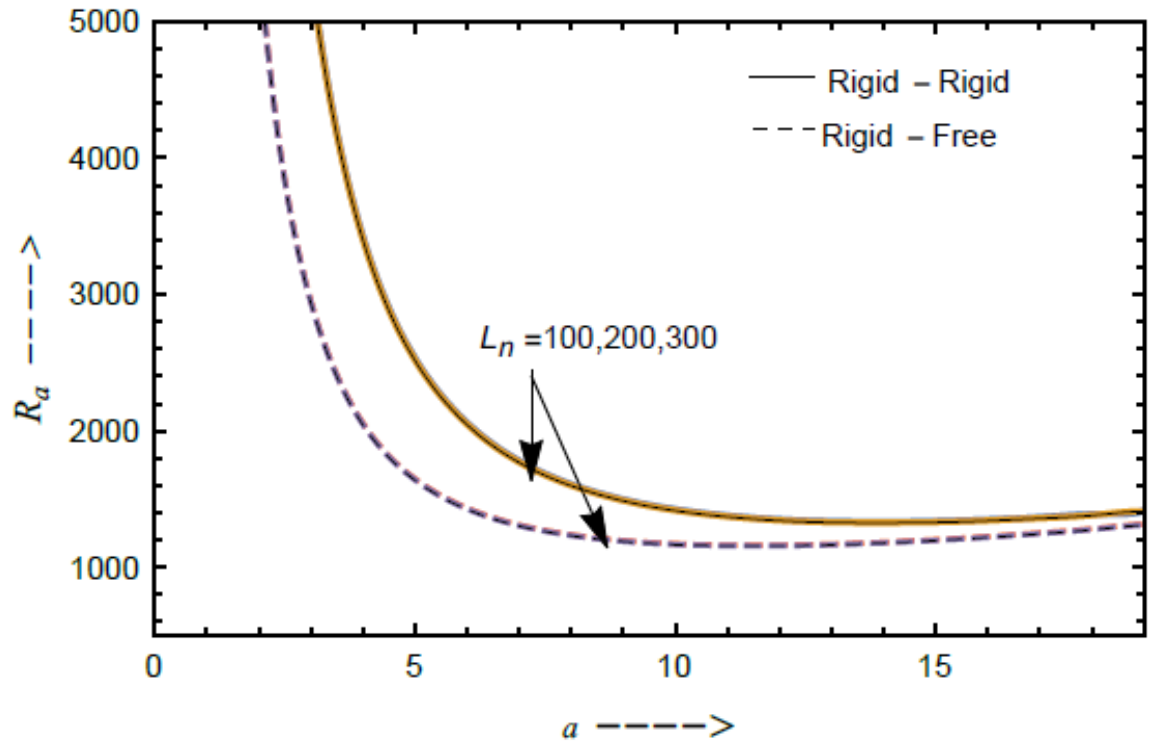

Figure 9. Influence of Lewis number on $R_{a}$ (osc) for $L_{n}=100,200$ and 300 for three different boundaries 
International Journal of Mathematical, Engineering and Management Sciences

Vol. 4, No. 1, 139-156, 2019

https://dx.doi.org/10.33889/IJMEMS.2019.4.1-013

Figures 6-7 show the variation of Rayleigh number $R_{a}($ stat $)$ and $R_{a}(\mathrm{osc})$ for $\varepsilon=0.5,0.6$ and 0.7, respectively for rigid-rigid and rigid-free boundaries. As shown for alumina-water nanofluid, increase in porosity hastens the onset of oscillatory as well as non-oscillatory convection.

Figures 8-9 show the impact of Lewis number on the convection problem for $L_{n}=100,200$ and 300 . It has been observed that with the rise in $L_{n}, R_{a}($ stat $)$ increases predominantly and $R_{a}(\mathrm{osc})$ does not show any significant change. Both rigid boundaries are more stable as compared to rigid-free boundaries.

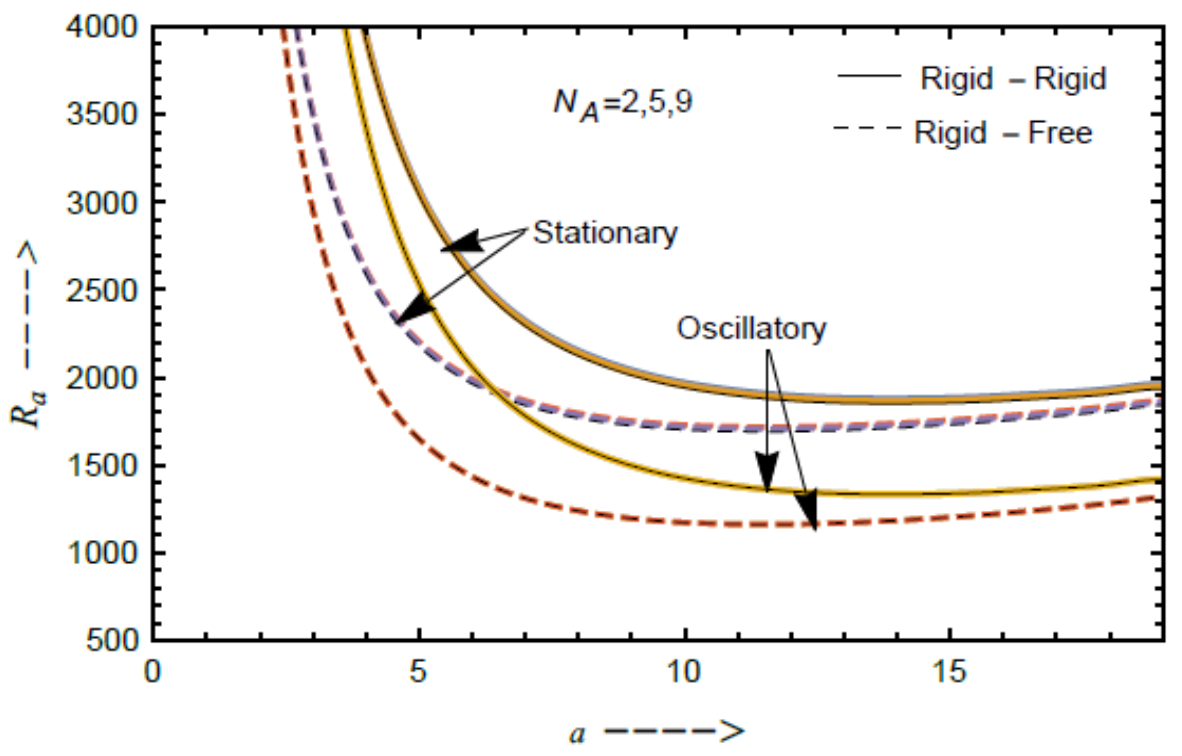

Figure 10. Influence of modified diffusivity ratio on $R_{a}$ (stat) and $R_{a}(\mathrm{osc})$ for $N_{A}=2,5$ and 9 for three different boundaries 
International Journal of Mathematical, Engineering and Management Sciences

Vol. 4, No. 1, 139-156, 2019

https://dx.doi.org/10.33889/IJMEMS.2019.4.1-013

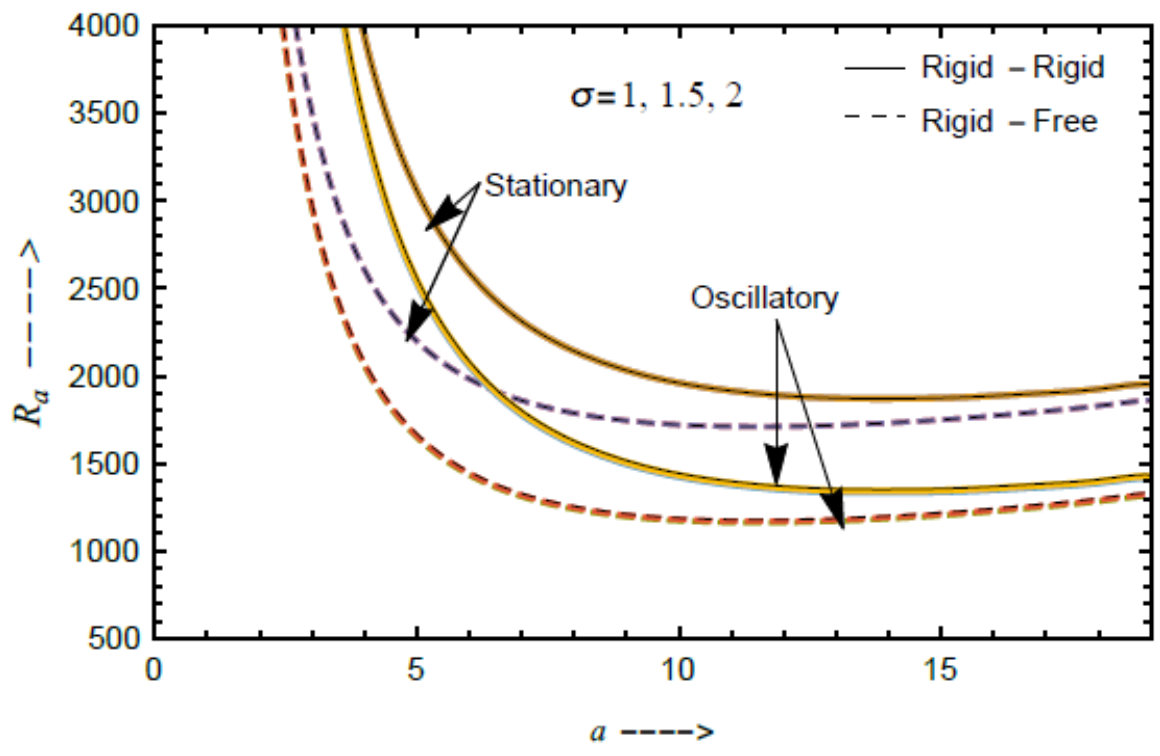

Figure 11. Influence of heat capacity ratio on $R_{a}($ stat $)$ and $R_{a}(\mathrm{osc})$ for $\sigma=1,1.5$ and 2 for three different boundaries

Figure 10 depicts the variation of $R_{a}$ (stat) and $R_{a}(\mathrm{osc})$ for $N_{A}=2,5$ and 9 for the two boundary conditions. In addition to the observation that the mode of instability is overstability, it is found that $R_{a}($ stat $)$ and $R_{a}(\mathrm{osc})$ are independent of the effect of diffusivity ratio. Figure 11 corresponds to $R_{a}$ (stat) and $R_{a}(\mathrm{osc})$ for $\sigma=1,1.5$ and $2 . R_{a}$ (stat) is independent of the heat capacity ratio and $R_{a}$ (osc) slightly rises with the rise in $\sigma$ (not seen in graphs). This is due to the fact that Eqs. (37), (52) are (57) are independent of the heat capacity ratio. The convection sets in through oscillatory motions and $R_{a}(\mathrm{osc})$ for both rigid boundaries is much more than that for rigid-free boundaries.

\section{Conclusions}

The present article investigates Rayleigh-Bénard convection for nanofluids under the influence of magnetic field for more realistic boundary conditions: rigid-free and rigid-rigid. The solution of the eigen value problem is found in terms of Rayleigh number by implementing the technique of normal modes and weighted residual Galerkin approximation. It has been found that the mode of heat transfer is through oscillatory motions. It is expected as buoyancy forces are acting oppositely. The critical values of wave number and Rayleigh number are found for alumina-water nanofluid. It is observed that $R_{c}$ shows a predominant fall with the increase in the ratio of pore space to total space for all the boundaries. As one moves from rigid-free boundaries to both rigid boundaries $a_{c}$ shows an appreciable increase along with moderate increase in $R_{c}$. Both $a_{c}$ and $R_{c}$ exhibit a substantial increase as the Lorentz force is increased which shows that magnetic field strongly delays the onset of thermal convection. When magnetic field and nanoparticles are not present in the nanofluid layer, $a_{c}$ and $R_{c}$ for rigid-free boundaries are found to be 3.97 and 56.97, respectively, which lie below the critical wave number and critical Rayleigh number for both 
International Journal of Mathematical, Engineering and Management Sciences

Vol. 4, No. 1, 139-156, 2019

https://dx.doi.org/10.33889/IJMEMS.2019.4.1-013

rigid boundaries $\left(a_{c}=4.791, R_{c}=72.94\right.$ ) for fixed value of porosity $\varepsilon=0.7$. The nanofluid layer system with both rigid boundaries is comparatively stable than rigid-free boundaries. To analyse the behavior of parameters like $R_{c n}, Q, \varepsilon, L_{n}, N_{A}$ and $\sigma$ computations are carried out using the software Mathematica to find $R_{a}$ (stat) and $R_{a}$ (osc). When the nanoparticle density decreases upward; the concentration Rayleigh number makes the stationary convection much more stable as compared to over stable motions. Lewis number is found to have dual character stabilizing for stationary convection and destabilizing for the oscillatory mode. It is also found that $R_{a}($ stat $)$ and $R_{a}$ (osc) are independent of the diffusivity ratio. As far as the effect of heat capacity ratio is concerned $R_{a}($ stat $)$ is independent of it whereas $R_{a}(\mathrm{osc})$ shows a slight rise with the rise in heat capacity ratio.

\section{Appendix B1: Expression for $\boldsymbol{R}_{\boldsymbol{a}}$ (osc) for Rigid-rigid Boundaries}

Separating the real and imaginary parts of Eq.(52) after putting $n=i \omega \neq 0$, we get an expression of $R_{a}(\mathrm{osc})$ for the rigid-rigid boundaries as

$$
\begin{aligned}
R_{a}(\mathrm{osc})= & {\left[141120 L_{n} \operatorname{Pr}_{1} Q \sigma+17472 L_{n} \operatorname{Pr}_{1} Q a^{2} \sigma+270 L_{n} \operatorname{Pr}_{2} R_{c n} a^{2} \sigma+336 L_{n} \operatorname{Pr}_{1} Q a^{4} \sigma\right.} \\
+ & 27 L_{n} \operatorname{Pr}_{2} R_{c n} a^{4} \sigma+141120 L_{n} \operatorname{Pr}_{1} \varepsilon \sigma+33600 \operatorname{Pr}_{2} \varepsilon \sigma+20832 L_{n} \operatorname{Pr}_{1} a^{2} \varepsilon \sigma+9520 \operatorname{Pr}_{2} a^{2} \varepsilon \sigma \\
& -270 N_{A} \operatorname{Pr}_{2} R_{c n} a^{2} \varepsilon \sigma+952 L_{n} \operatorname{Pr}_{1} a^{4} \varepsilon \sigma+896 \operatorname{Pr}_{2} a^{4} \varepsilon \sigma-27 N_{A} \operatorname{Pr}_{2} R_{c n} a^{4} \varepsilon \sigma+28 L_{n} \operatorname{Pr}_{1} a^{6} \varepsilon \sigma \\
& +28 \operatorname{Pr}_{2} a^{6} \varepsilon \sigma+141120 \operatorname{Pr}_{1} Q \sigma^{2}+17472 \operatorname{Pr}_{1} Q a^{2} \sigma^{2}+252 L_{n} \operatorname{Pr}_{1} R_{c n} a^{2} \sigma^{2}+336 \operatorname{Pr}_{1} Q a^{4} \sigma^{2} \\
& +27 L_{n} \operatorname{Pr}_{1} R_{c n} a^{4} \sigma^{2}+141120 \operatorname{Pr}_{1} \varepsilon \sigma^{2}+20832 \operatorname{Pr}_{1} a^{2} \varepsilon \sigma^{2}+952 \operatorname{Pr}_{1} a^{4} \varepsilon \sigma^{2}+28 \operatorname{Pr}_{1} a^{6} \varepsilon \sigma^{2} \\
& \left.-336 L_{n} \operatorname{Pr}_{2} \varepsilon \omega^{2}-28 L_{n} \operatorname{Pr}_{2} a^{2} \varepsilon \omega^{2}\right]\left[\left(9 a^{2} \varepsilon \sigma\right)\left(28 L_{n} \operatorname{Pr}_{1}+30 \operatorname{Pr}_{2}+3 L_{n} \operatorname{Pr}_{1} a^{2}+3 \operatorname{Pr}_{2} a^{2}\right)\right]^{-1},
\end{aligned}
$$

where $\omega^{2}=\frac{-X_{2}+\sqrt{X_{2}^{2}-4 X_{1} X_{3}}}{2 X_{1}}$, which is obtained by eliminating $R_{a}$ (osc) between real and imaginary parts of the eigen value Eq. (52) and $X_{1}, X_{2}, X_{3}$ are the coefficients of the biquadratic equation $X_{1} \omega^{4}+X_{2} \omega^{2}+X_{3}=0$.

\section{Appendix B2: Expression for $\boldsymbol{R}_{a}$ (osc) for Rigid-Free Boundaries}

In this case the eigen value Eq. (57) gives the expression for $R_{a}$ (osc) by separating real and imaginary parts as 
International Journal of Mathematical, Engineering and Management Sciences

Vol. 4, No. 1, 139-156, 2019

https://dx.doi.org/10.33889/IJMEMS.2019.4.1-013

$$
\begin{aligned}
R_{a}(\mathrm{osc})= & {\left[1270080 L_{n} \operatorname{Pr}_{1} Q \sigma+187488 L_{n} \operatorname{Pr}_{1} Q a^{2} \sigma+5070 L_{n} \operatorname{Pr}_{2} R_{c n} a^{2} \sigma+6048 L_{n} \operatorname{Pr}_{1} Q a^{4} \sigma\right.} \\
+ & 507 L_{n} \operatorname{Pr}_{2} R_{c n} a^{4} \sigma+1270080 L_{n} \operatorname{Pr}_{1} \varepsilon \sigma+604800 \operatorname{Pr}_{2} \varepsilon \sigma+247968 L_{n} \operatorname{Pr}_{1} a^{2} \varepsilon \sigma+ \\
& 174160 \operatorname{Pr}_{2} a^{2} \varepsilon \sigma-5070 N_{A} \operatorname{Pr}_{2} R_{c n} a^{2} \varepsilon \sigma+17416 L_{n} \operatorname{Pr}_{1} a^{4} \varepsilon \sigma+16688 \operatorname{Pr}_{2} a^{4} \varepsilon \sigma \\
& -507 N_{A} \operatorname{Pr}_{2} R_{c n} a^{4} \varepsilon \sigma+532 L_{n} \operatorname{Pr}_{1} a^{6} \varepsilon \sigma+532 \operatorname{Pr}_{2} a^{6} \varepsilon \sigma+1270080 \operatorname{Pr}_{1} Q \sigma^{2} \\
+ & 187488 \operatorname{Pr}_{1} Q a^{2} \sigma^{2}+4914 L_{n} \operatorname{Pr}_{1} R_{c n} a^{2} \sigma^{2}+6048 \operatorname{Pr}_{1} Q a^{4} \sigma^{2}+507 L_{n} \operatorname{Pr}_{1} R_{c n} a^{4} \sigma^{2} \\
+ & 1270080 \operatorname{Pr}_{1} \varepsilon \sigma^{2}+247968 \operatorname{Pr}_{1} a^{2} \varepsilon \sigma^{2}+17416 \operatorname{Pr}_{1} a^{4} \varepsilon \sigma^{2}+532 \operatorname{Pr}_{1} a^{6} \varepsilon \sigma^{2} \\
& \left.-6048 L_{n} \operatorname{Pr}_{2} \varepsilon \omega^{2}-532 L_{n} \operatorname{Pr}_{2} a^{2} \varepsilon \omega^{2}\right]\left[\left(36 L_{n} a^{2} \varepsilon \sigma\right)\left(126 \operatorname{Pr}_{1}+130 \operatorname{Pr}_{2}+13 \operatorname{Pr}_{1} a^{2}+13 \operatorname{Pr}_{2} a^{2}\right)\right]^{-1}
\end{aligned}
$$

where $\omega^{2}=\frac{-X_{2}+\sqrt{X_{2}^{2}-4 X_{1} X_{3}}}{2 X_{1}}$, is obtained in the same manner as for rigid-rigid boundaries and $X_{1}, X_{2}, X_{3}$ are the coefficients of the biquadratic equation $X_{1} \omega^{4}+X_{2} \omega^{2}+X_{3}=0$.

\section{Conflict of Interest}

The authors confirm that there is no conflict of interest to declare for this publication.

\section{Acknowledgement}

The research work presented in this chapter has been supported by grants to second author from CSIR, New Delhi-110012, India via Project [Ref. No: 25(0247)/15/EMR-II].

\section{References}

Bhadauria, B. S., \& Agarwal, S. (2011). Natural convection in a nanofluid saturated rotating porous layer: A Nonlinear study. Transport in Porous Media, 87(2), 585-602.

Buongiorno, J. (2006). Convective transport in nanofluids. ASME Journal of Heat Transfer, 128(3), 240250.

Buongiorno, J. and Hu, W. (2005). Nanofluid coolants for advanced nuclear power plants. Paper No. 5705, Proceedings of ICAPP'05, Seoul.

Chandrasekhar, S. (1981). Hydrodynamic and hydromagnetic stability. New York: Dover Publications.

Choi, S. (1995). Enhancing thermal conductivity of fluids with nanoparticles: Development and Applications of Non-Newtonian flows. ASME FED- 231/MD-vol. 66, 99-105.

Das, S. K., Putra, N., Thiesen, P., \& Roetzel, W. (2003). Temperature dependence of thermal conductivity enhancement for nanofluids. ASME Journal of Heat Transfer, 125(4), 567-574.

Gupta, U., \& Ahuja, J. (2014). Hall Effect on thermal convection of a nanofluid layer saturating a porous medium. International Journal of Technology, 4(1), 214-219.

Gupta, U., Ahuja, J., \& Wanchoo, R. K. (2013). Magneto convection in a nanofluid layer. International Journal of Heat and Mass Transfer, 64, 1163-1171. 
International Journal of Mathematical, Engineering and Management Sciences

Vol. 4, No. 1, 139-156, 2019

https://dx.doi.org/10.33889/IJMEMS.2019.4.1-013

Heris, S. Z., Salehi, H., \& Noie, S. H. (2012). The effect of magnetic field and nanofluid on thermal performance of two phase closed thermosyphon. International Journal of Physical Sciences, 7(4), 534-543.

Kim, J., Kang, Y. T., \& Choi, C. K. (2004). Analysis of convective instability and heat transfer characteristics of nanofluids. Physics of Fluids, 16(7), 2395-2401.

Kleinstreuer, C., Li. J., \& Koo, J. (2008). Microfluidics of nano-drug delivery. International Journal of Heat and Mass Transfer, 51(23-24), 5590-5597.

Masuda, H., Ebata, A., Teramae, K., \& Hishinuma, N. (1993). Alteration of thermal conductivity and viscosity of liquid by dispersing ultra fine particles. Netsu Bussei, 7(4), 227-233.

Nield, D. A., \& Kuznetsov, A. V. (2009). Thermal instability in a porous medium layer saturated by a nanofluid. International Journal of Heat and Mass Transfer, 52(25-26), 5796-5801.

Nield, D. A., \& Kuznetsov, A. V. (2010). The onset of convection in a horizontal nanofluid layer of finite depth. European Journal of Mechanics- B/Fluids, 29(3), 217-223.

Tzou, D.Y. (2008). Instability of nanofluids in natural convection. ASME Journal of Heat Transfer, 130(7), 372-401.

Yadav, D., Agrawal, G. S., Bhargava, R. (2011). Thermal instability of rotating nanofluid layer. International Journal of Engineering Science, 49(11), 1171-1184. 